\title{
Aloperine attenuates carbon tetrachloride-induced mouse hepatic injury via Nrf2/HO-1 pathway
}

\author{
Rui Xiong ${ }^{1}$, Shuzhong Shan ${ }^{2 *}$, Xiaoming Wang ${ }^{1}$, Xiaowen Zhang ${ }^{1}$, Haixia $Y u^{1}$, \\ Haomin Shi ${ }^{1}$, Xuejiao Wang ${ }^{1}$ \\ ${ }^{1}$ Department of Clinical Nutrition, ${ }^{2}$ Department of Orthopaedics, Qinhai Provincial People's Hospital, Xining City, Qinghai \\ Province 810007, China \\ *For correspondence: Email: ShuzhongShandjk@163.com; Tel: +86-971-8066178
}

Sent for review: 10 February 2020

Revised accepted: 24 April 2020

\begin{abstract}
Purpose: To investigate whether aloperine pretreatment ameliorates acute liver injury in carbon tetrachloride $\left(\mathrm{CCl}_{4}\right)$-treated mice.

Methods: Mice were injected with $\mathrm{CCl}_{4}$ and orally administered aloperine. Blood samples and liver tissues were used for histopathological and biochemical analyses, respectively. Protein expression levels were determined by western blotting.

Results: Histopathological analysis indicate that aloperine pretreatment significantly alleviated $\mathrm{CCl}_{4-}$ induced mouse hepatic injury. $\mathrm{CCl}_{4}$ treatment induced the upregulation of aspartate aminotransferase $(A S T)$, alkaline phosphatase $(A L P)$, alanine amino transferase $(A L T)$, and total bilirubin $(p<0.05)$. However, these alterations were significantly inhibited by aloperine treatment. Moreover, aloperine pretreatment markedly decreased $(p<0.05)$ the $\mathrm{CCl}_{4}$-induced expression of oxidative stress biomarkers, including malondrialdeline (MDA), glutathione (GSH), catalase (CAT), and superoxide dismutase (SOD). Compared to the control group, the protein levels of Nrf2, HO-1, iNOS, and COX-2 were significantly increased in the $\mathrm{CCl}_{4}$ group, while $\mathrm{Nrf2}$ and $\mathrm{HO}-1$ were upregulated. Furthermore, iNOS and COX-2 were downregulated in mouse liver in $\mathrm{CCl}_{4}+$ aloperine group compared to $\mathrm{CCl}_{4}$ group in a concentration-dependent manner $(p<0.05)$.

Conclusion: Aloperine pretreatment appears to markedly upregulate Nrf2 and HO-1 and downregulate iNOS and COX-2 to suppress hepatic injury in mice. Thus, aloperine is a promising treatment for acute liver injury.
\end{abstract}

Keywords: Hepatic injury, Aloperine, Oxidative stress, Nrf2/HO-1 pathway

\begin{abstract}
This is an Open Access article that uses a fund-ing model which does not charge readers or their institutions for access and distributed under the terms of the Creative Commons Attribution License (http://creativecommons.org/licenses/by/4.0) and the Budapest Open Access Initiative (http://www.budapestopenaccessinitiative.org/read), which permit unrestricted use, distribution, and reproduction in any medium, provided the original work is properly credited.
\end{abstract}

Tropical Journal of Pharmaceutical Research is indexed by Science Citation Index (SciSearch), Scopus, International Pharmaceutical Abstract, Chemical Abstracts, Embase, Index Copernicus, EBSCO, African Index Medicus, JournalSeek, Journal Citation Reports/Science Edition, Directory of Open Access Journals (DOAJ), African Journal Online, Bioline International, Open-J-Gate and Pharmacy Abstracts

\section{INTRODUCTION}

Environmental toxins often disturb hepatic metabolic function and increase the expression of liver enzymes, leading to liver fibrosis, cirrhosis, and even cancer [1]. Liver injury can be caused by a combination of oxidative stress, necrosis, inflammation, and apoptosis [2]. Carbon tetrachloride $\left(\mathrm{CCl}_{4}\right)$ is frequently used to induce liver injury and to study the effects of chemical compounds on the liver [3]. Existing hepatoprotective drugs are used sparingly 
because of their side effects [4]. Therefore, there is an urgent need to develop safer hepatoprotective drugs.

Nuclear factor erythroid 2-related factor 2 (Nrf2) is an important leucine zipper-containing, redoxsensitive transcription factor that can induce the production of antioxidant enzymes via the antioxidant response element [5]. By activating heme oxygenase-1 (HO-1) expression, Nrf2 can suppress the nuclear translocation of NF-kB and subsequent inflammatory responses [6]. Thus, interventions that target the $\mathrm{Nrf} 2 / \mathrm{HO}-1$ signaling pathway have recently been suggested to be important therapeutic approaches for the treatment of alcoholic liver injury [7]. Antioxidants not only remove free radicals, but are also cytoprotective [8]. For example, the combination of metformin and luteolin has been reported to reduce liver injury induced by $\mathrm{CCl}_{4}$ by activating the Nrf2/HO-1 pathway [9]. The Nrf2/HO-1 pathway thus appears vital for treating liver injury.

Aloperine, a compound that can be extracted from bitter beans, plays an important role in certain inflammatory disorders, such as allergic contact dermatitis and experimental colitis $[10,11]$. A recent study showed that aloperine protects mice from ischemia-reperfusion-induced renal injury by reducing levels of oxidative stress [12]. Furthermore, aloperine was found to attenuate allergic airway inflammation by regulating the Nrf2/HO-1 signaling pathway [13]. Therefore, whether aloperine could alleviate liver injury remains to be investigated. The present study was undertaken to explore the influence of aloperine pretreatment on acute liver injury caused by $\mathrm{CCl}_{4}$ in mice and determine the possible involvement of the Nrf2/HO-1 pathway in this process.

\section{EXPERIMENTAL}

\section{Chemical and reagents}

Aloperine and $\mathrm{CCl}_{4}$ were purchased from Abcam (Cambridge, MA, USA) and Sigma-Aldrich (St. Louis, MO, USA), respectively.

\section{Mice and treatments}

The animal study was approved the Medical Ethics Committee of Qinhai Provincial People's Hospital (approval no. 20191016) and conducted according to the Guidelines for the Care and Use of Laboratory Animals published by the National Institutes of Health [14]. Twenty-four male C57BL/6 mice (6-8 weeks of age, 16-18 g; Animal Research Center of Nanjing University
(Nanjing, China) were randomly divided into four groups (six mice/group): Control, $\mathrm{CCl}_{4}, \mathrm{CCl}_{4}+50$ $\mathrm{mg} / \mathrm{kg}$ aloperine, and $\mathrm{CCl}_{4}+100 \mathrm{mg} / \mathrm{kg}$ aloperine. In the $\mathrm{CCl}_{4}$ group, mice were intraperitoneally (i.p.) injected with $\mathrm{CCl}_{4} \quad[10$ $\mathrm{mL} / \mathrm{kg}, 0.5 \%(\mathrm{v} / \mathrm{v})$, dissolved in olive oil]. In the $\mathrm{CCl}_{4}+50 \mathrm{mg} / \mathrm{kg}$ aloperine and $\mathrm{CCl}_{4}+100$ aloperine groups, mice were orally administered aloperine (50 and $100 \mathrm{mg} / \mathrm{kg}$, respectively, resuspended in ethanol) for 7 days. Two hours after the last dose of aloperine, mice were injected with $\mathrm{CCl}_{4}$. Mice in the control and $\mathrm{CCl}_{4}$ groups were given the same dose of solvent. Mice were sacrificed $24 \mathrm{~h}$ post-CCl 4 injection. Blood samples were taken from the orbital vein, while liver tissues were obtained after dissection and used for the following analyses.

\section{Hepatic histopathological examination}

Liver tissues were fixed, embedded, and stained with hematoxylin and eosin (H\&E). The extent of injury was scored from 0 to 4 , based on the severity of the vacuolization of hepatocyte cytoplasm, sinusoidal congestion, and parenchymal necrosis [15]. Ten different views of an image were randomly chosen and scored by two pathologists independently.

\section{Evaluation of biomarkers of liver function}

Serum AST, ALP ALT, and total bilirubin levels were measured utilizing commercial assay kits (Jiancheng Biological Technology, China).

\section{Antioxidants in hepatic tissues}

Hepatic tissues were homogenized and centrifuged. The supernatants of the liver tissue homogenates were used for the determination of malondrialdeline (MDA), superoxide dismutase (SOD), catalase (CAT), and glutathione (GSH) levels. Commercial assay kits (Jiancheng Biological Technology) were used to examine the effects of the antioxidants on hepatic tissues.

\section{Protein extraction and western blotting analysis}

Western blotting was conducted, as described previously $[16,17]$. Total protein was extracted, subjected to SDS-PAGE, and then transferred onto PVDF membranes. The membranes were then blocked with $5 \%$ nonfat milk and incubated with the following antibodies: anti-Nrf2 (Abcam); anti-HO-1(Cell Signaling Technology, Danvers, MA, USA), anti-iNOS (Abcam); and anti-COX-2 (Abcam). The level of $\beta$-actin was used as an internal control. Proteins were visualized and quantified. 


\section{Statistical analysis}

The results are expressed as mean $\pm S D$ and analyzed by one-way ANOVA among the four groups using SPSS software. $P<0.05$ was considered statistically significant.

\section{RESULTS}

\section{Aloperine attenuates $\mathrm{CCl}_{4}$-induced liver injury in mice}

To verify the role of aloperine in reducing hepatic damage, liver tissues were collected from mice in the different groups and examined by histological analysis and scored. Compared to control mice, $\mathrm{CCl}_{4}$ treatment resulted in severe liver damage (Figure 1). The hepatic damage was alleviated to a certain extent by treatment with $50 \mathrm{mg} / \mathrm{kg}$ aloperine, but was ameliorated to a much greater extent by treatment with $100 \mathrm{mg} / \mathrm{kg}$ aloperine. These findings demonstrate that aloperine reduces $\mathrm{CCl}_{4}$-induced liver damage in mice.
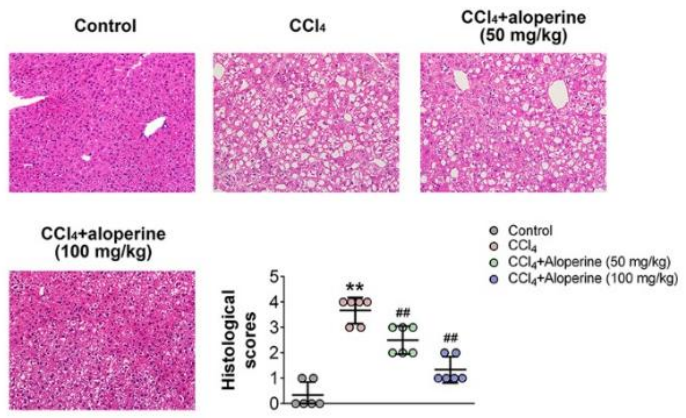

Figure 1: Morphological and histological features of liver tissues from mice in the different groups. H\&E staining of liver sections from the various mouse groups was performed. Scores were assigned, according to the severity of liver injury, and are shown as a scatter diagram. Original magnification, 200x. **/\#\#, $P<0.01$

\section{Aloperine treatment decreases $\mathrm{CCl}_{4}$-induced serum levels of hepatic functional markers in mice}

The effects of aloperine on the serum levels of hepatic functional markers were determined. Compared to the control group, the serum levels of AST, ALT, ALP, and total bilirubin were significantly increased in mice by $\mathrm{CCl}_{4}$ treatment. However, aloperine treatment $(50 \mathrm{mg} / \mathrm{kg})$ markedly reduced the levels of these hepatic functional markers compared to mice with $\mathrm{CCl}_{4}$ treatment. Moreover, the serum concentrations of these markers were lower in the $\mathrm{CCl}_{4}+100$ $\mathrm{mg} / \mathrm{kg}$ aloperine group than in the $\mathrm{CCl}_{4}+50$ $\mathrm{mg} / \mathrm{kg}$ aloperine group (Figure 2).

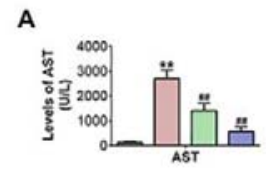

B

C
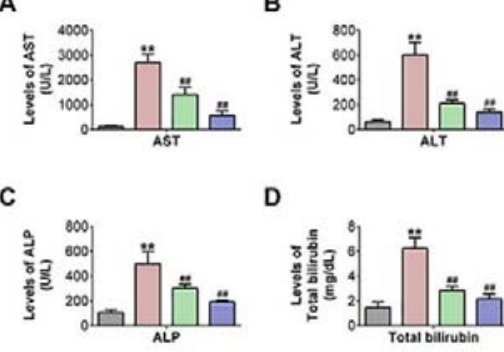

D

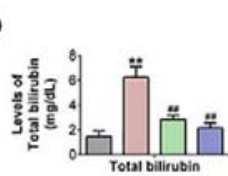

Figure 2: Effect of aloperine on the serum levels of hepatic functional markers in mice. ${ }^{* *} / \# \#, P<0.01$

\section{Aloperine treatment reduces $\mathrm{CCl}_{4}$-induced oxidative stress in mouse liver}

$\mathrm{CCl}_{4}$ treatment for $24 \mathrm{~h}$ markedly upregulated the levels of MDA and downregulated the activities of $\mathrm{GSH}, \mathrm{CAT}$, and SOD. Aloperine pretreatment reversed these effects in a dose-dependent manner (Figure 3).

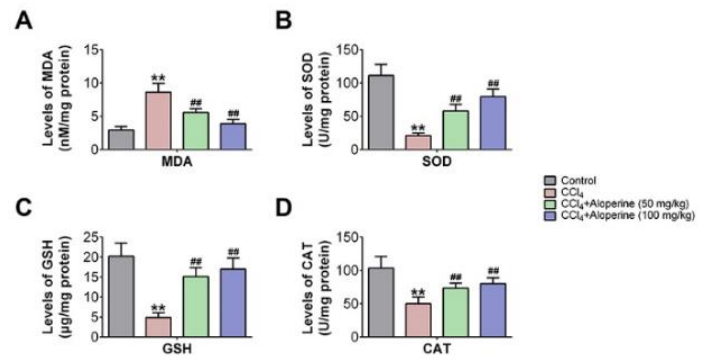

Figure 3: Effects of aloperine on $\mathrm{CCl}_{4}$-induced oxidative stress in mouse liver. ${ }^{* *} / \# \#, P<0.01$.

Aloperine reverses the effects of $\mathrm{CCl}_{4}$ treatment on the regulation of the Nrf2/HO-1 axis in mouse liver

The results shown in Figure 4 showed that, compared to the control group, the protein levels of Nrf2, HO-1, iNOS, and COX-2 were significantly increased in the $\mathrm{CCl}_{4}$ group. On the other hand, Nrf2 and HO-1 were upregulated, whereas iNOS and COX-2 were downregulated in mouse liver in $\mathrm{CCl}_{4}+$ aloperine group compared to the $\mathrm{CCl}_{4}$ group in a concentrationdependent manner.

\section{DISCUSSION}

The liver has numerous important functions that are essential for life, including protein synthesis, glucose homeostasis, and detoxification. Although the liver has a strong regenerative capacity, it can still become damaged due to exposure environmental toxins, resulting in organ 
dysfunction and metabolic abnormalities. The underlying molecular mechanism of acute liver injury has been found to be associated with oxidative stress, apoptosis, and inflammation [1]. $\mathrm{CCl}_{4}$ treatment is known to stimulate lipid peroxidation, reactive oxygen species production, and centrilobular necrosis and steatosis; it has been extensively used to induce acute hepatic damage in a mouse model of liver toxicity [18].

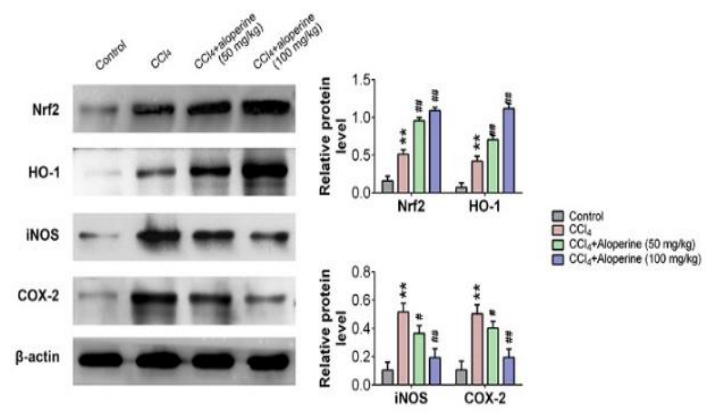

Figure 4: Effects of aloperine treatment on the expression of $\mathrm{Nrf} 2 / \mathrm{HO}-1$ pathway components in mouse liver. Relative protein levels are shown in the western blot. \#, $P<0.05,{ }^{\star *} / \# \#, P<0.01$

Some natural products have been shown to relieve $\mathrm{CCl}_{4}$-induced liver damage. For example, curcumin, an extract from turmeric rhizomes, can inhibit oxidative stress and inflammation, ameliorating $\mathrm{CCl}_{4}$-induced liver injury [19]. In addition, sesamin has been suggested to regulate the JNK pathway and inhibit hepatic oxidative stress induced by $\mathrm{CCl}_{4}$ [20]. In the present study, aloperine treatment effectually relieved hepatic dysfunction and histopathologic damage by downregulating serum AST, ALT, and ALP activities, and total levels of bilirubin. The combination of two or more agents may result in synergistic effects to alleviate hepatic injury, which could potentially lessen the dose of a single drug and reduce untoward side effects. For example, the combination of metformin and luteolin has been shown to exhibit a potential synergy for the treatment of hepatic injury mediated by $\mathrm{CCl}_{4}$ [9]. Whether these medications can be used in combination with aloperine to prevent liver damage deserves further study.

Aloperine has been found to possess some beneficial medicinal functions. It protects mice against DSS-induced colitis via the suppression of the PI3K/Akt signaling pathway [21]. Aloperine pretreatment has also been shown to reduce inflammation, the apoptosis of tubular cells, and renal damage caused by ischemia-reperfusion [12]. Furthermore, aloperine was shown to inhibit the proliferation and differentiation of fibroblasts, alleviating lung fibrosis induced by bleomycin
[22]. The present study demonstrated that aloperine could inhibit oxidative stress responses and elicit hepatoprotective effects. Based on these findings, aloperine may have other medicinal benefits that should be explored in the future.

Nrf2 has been shown to regulate antioxidant genes; its activation might provide effective cellular protection by regulating intracellular redox status [8]. Previous studies have suggested that natural antioxidants may activate Nrf2 and HO-1. For example, curcumin, an activator of the Nrf2 pathway, was shown to stimulate the activity of antioxidants and suppress the oxidative stress caused by $\mathrm{CCl}_{4}$ [19]. In addition, morin [23], anwulignan [24], and andrographolide [25] have been shown to upregulate $\mathrm{Nrf} 2 / \mathrm{HO}-1$, exert antioxidative functions, and protect against mouse liver damage. Therefore, the stimulation of the Nrf2/HO-1 pathway is a promising strategy for the clinical treatment of liver injury.

\section{CONCLUSION}

The findings of this study indicate that aloperine pretreatment suppresses mouse hepatic injury, partly via the enhancement of $\mathrm{Nrf} 2 / \mathrm{HO}-1$ pathway and stimulation of its antioxidant activity. These findings may thus lead to the development of novel drug candidates for the management of acute liver injury.

\section{DECLARATIONS}

\section{Acknowledgement}

This work was supported by Natural Science Foundation of Qinghai Province (Grant no. 2018ZJ-715).

\section{Conflict of interest}

No conflict of interest is associated with this work.

\section{Contribution of authors}

We declare that this work was done by the authors named in this article and all liabilities pertaining to claims relating to the content of this article will be borne by the authors.

\section{Open Access}

This is an Open Access article that uses a funding model which does not charge readers or their institutions for access and distributed under the 
terms of the Creative Commons Attribution License (http://creativecommons.org/licenses/by/ 4.0) and the Budapest Open Access Initiative (http://www.budapestopenaccessinitiative.org/rea d), which permit unrestricted use, distribution, and reproduction in any medium, provided the original work is properly credited.

\section{REFERENCES}

1. Jaeschke H, McGill MR, Ramachandran A. Oxidant stress, mitochondria, and cell death mechanisms in drug-induced liver injury: Lessons learned from acetaminophen hepatotoxicity. Drug Metab Rev 2012; 44(1): 88-106.

2. Cong $M$, Zhao WH, Liu TH, Wang P, Fan X, Zhai QL, Bao XL, Zhang $D$, You $H$, Kisseleva $T$ et al. Protective effect of human serum amyloid $P$ on CCl4-induced acute liver injury in mice. Int $\mathrm{J} \mathrm{Mol}$ Med 2017; 40(2): 454-464.

3. Tian $X T$, Liu $H$, Qiao $S D$, Yin $H$, Chen MC, Hu P, Wang YY, Peng HG, Liu F, Pan GY et al. Exploration of the hepatoprotective chemical base of an orally administered herbal formulation (YCHT) in normal and CCl4-intoxicated liver injury rats. Part 2: Hepatic disposition in vivo and hepatoprotective activity in vitro. J Ethnopharmacol 2019; 236: 161-172.

4. Yousefi-Manesh H, Shirooie S, Partoazar A, Nikoui V, Estakhri MRA, Bakhtiarian A. Hepatoprotective effects of phosphatidylserine liposomes on carbon tetrachloride-induced hepatotoxicity in rats. J Cell Biochem 2019; 120(7): 11853-11858.

5. Boettler U, Volz N, Teller N, Haupt LM, Bakuradze T, Eisenbrand G, Bytof G, Lantz I, Griffiths LR, Marko D. Induction of antioxidative Nrf2 gene transcription by coffee in humans: depending on genotype? Mol Biol Rep 2012; 39(6): 7155-7162.

6. Bellezza I, Tucci A, Galli F, Grottelli S, Mierla AL, Pilolli F, Minelli A. Inhibition of NF-kappa B nuclear translocation via HO-1 activation underlies alpha-tocopheryl succinate toxicity. J Nutr Biochem 2012; 23(12): 1583-1591.

7. Kumar KJS, Liao JW, Xiao JH, Vani MG, Wang SY. Hepatoprotective effect of lucidone against alcoholinduced oxidative stress in human hepatic HepG2 cells through the up-regulation of $\mathrm{HO}-1 / \mathrm{Nrf}-2$ antioxidant genes. Toxicol in Vitro 2012; 26(5): 700-708.

8. Cao MN, Wang HX, Guo LM, Yang SM, Liu C, Khor TO, Yu SW, Kong AN. Dibenzoylmethane Protects Against CCl4-Induced Acute Liver Injury by Activating Nrf2 via JNK, AMPK, and Calcium Signaling. Aaps J 2017; 19(6): 1703-1714.

9. Yan Y, Jun C, Lu Y, Song JM. Combination of metformin and luteolin synergistically protects carbon tetrachlorideinduced hepatotoxicity: Mechanism involves antioxidant, anti-inflammatory, antiapoptotic, and Nrf2/HO-1 signaling pathway. Biofactors 2019; 45(4): 598-606.

10. Zhou Y, Wang H, Liang L, Zhao WC, Chen Y, Deng HZ. Total Alkaloids of Sophora alopecuroides Increases the
Expression of CD4(+) CD25(+) Tregs and IL-10 in Rats with Experimental Colitis. Am J Chinese Med 2010; 38(2): 265-277.

11. Yuan $X Y$, Liu W, Zhang $P$, Wang RY, Guo JY. Effects and mechanisms of aloperine on 2, 4dinitrofluorobenzene-induced allergic contact dermatitis in BALB/C mice. Eur J Pharmacol 2010; 629(1-3): 147152.

12. Hu S, Zhang $Y X$, Zhang $M$, Guo YC, Yang $P$, Zhang $S$, Simsekyilmaz $S, X u J F, L i J X$, Xiang $X D$ et al. Aloperine Protects Mice against Ischemia-Reperfusion (IR)Induced Renal Injury by Regulating PI3K/AKT/mTOR Signaling and AP-1 Activity. Mol Med 2015; 21:912-923.

13. Fu Q, Gao Y, Yang F, Mao T, Sun Z, Wang $H$, Song $B, L i$ $X$. Suppression of microRNA-454 impedes the proliferation and invasion of prostate cancer cells by promoting $\mathrm{N}$-myc downstream-regulated gene 2 and inhibiting WNT/3-catenin signaling. Biomed Pharmacother 2018; 97:120-127.

14. Kastenmayer RJ, Moore RM, Bright AL, Torres-Cruz R, Elkins WR. Select Agent and Toxin Regulations: Beyond the Eighth Edition of the Guide for the Care and Use of Laboratory Animals. J Am Assoc Lab Anim 2012; 51(3): 333-338.

15. Zhu RZ, Xiang D, Xie C, Li JJ, Hu JJ, He HL, Yuan YS, Gao J, Han W, Yu Y. Protective effect of recombinant human IL-1Ra on CCl4-induced acute liver injury in mice. World J Gastroentero 2010; 16(22): 2771-2779.

16. Zhu DP, Sun CX, Qian X. MST1 suppresses viability and promotes apoptosis of glioma cells via upregulating SIRT6 expression. J Integr Neurosci 2019; 18(2): 117126.

17. Fu ZY, Li YD, Yang SS, Ma CX, Zhao RR, Guo H, Wei $H P$. Angelica sinensis polysaccharide promotes apoptosis by inhibiting JAK/STAT pathway in breast cancer cells. Trop J Pharm Res 2019; 18(11): 22472253.

18. Kodai S, Takemura S, Minamiyama Y, Hai S, Yamamoto S, Kubo S, Yoshida Y, Niki E, Okada S, Hirohashi $K$ et al. S-allyl cysteine prevents CCl4-induced acute liver injury in rats. Free Radical Res 2007; 41(4): 489-497.

19. Peng XY, Dai CS, Liu QW, Li JK, Qiu JR. Curcumin Attenuates on Carbon Tetrachloride-Induced Acute Liver Injury in Mice via Modulation of the Nrf2/HO-1 and TGF1/Smad3 Pathway. Molecules 2018; 23(1).

20. Ma JQ, Ding J, Zhang L, Liu CM. Hepatoprotective properties of sesamin against CCl4 induced oxidative stress-mediated apoptosis in mice via JNK pathway. Food Chem Toxicol 2014; 64:41-48.

21. Fu $X X$, Sun F, Wang FX, Zhang JA, Zheng BY, Zhong $J X$, Yue TT, Zheng XB, XU JF, Wang CY. Aloperine Protects Mice against DSS-Induced Colitis by PP2AMediated PIJK/Akt/mTOR Signaling Suppression. Mediat Inflamm 2017.

22. Yin WL, Han J, Zhang ZJ, Han ZM, Wang SY. Aloperine Protects Mice against Bleomycin-induced Pulmonary Fibrosis by Attenuating Fibroblast Proliferation and Differentiation. Sci Rep-Uk 2018; 8

Trop J Pharm Res, May 2020; 19(5): 987 
23. Tian Y, Li Z, Shen BY, Zhang QL, Feng HH. Protective effects of morin on lipopolysaccharide/D-galactosamineinduced acute liver injury by inhibiting TLR4/NF-kappa $B$ and activating Nrf2/HO-1 signaling pathways. Int Immunopharmacol 2017; 45:148-155.

24. Gao JQ, Yu ZP, Jing S, Jiang $W H$, Liu $C, Y u C Y$, Sun JH, Wang CM, Chen JG, Li H. Protective effect of Anwulignan against $D$-galactose-induced hepatic injury through activating p38 MAPK-Nrf2-HO-I pathway in mice. Clin Interv Aging 2018; 13:1859-1869.

25. Pan CW, Yang SX, Pan ZZ, Zheng B, Wang JZ, Lu GR, Xue $Z X, X u$ CL. Andrographolide ameliorates $d$ galactosamine/lipopolysaccharide-induced acute liver injury by activating Nrf2 signaling pathway. Oncotarget 2017; 8(25): 41202-41210. 